\title{
Nurse aides' identification of onset and level of agitation in late stage dementia patients
}

\author{
Ann L. Whall, PhD, RN, FAAN \\ Margaret E. A. Black, PhD, RN \\ Dawn J. Yankou, PhD, RN \\ Carla J. Groh, PhD, RN \\ Barbara J. Kupferschmid, MSN, RN \\ Norman L. Foster, MD \\ Roderick Little, $\mathrm{PhD}$
}

\begin{abstract}
Nurse aides provide the majority of care to patients in nursing homes and thus are vital links in the early identification and treatment of agitation in dementia. Agitation increases in frequency as dementia progresses and unrecognized and untreated agitation may develop into a state of acute aggression (termed catastrophic reaction) in which demented patients become severely disturbed and may harm themselves or others. Nurse aides, however, are sometimes characterized as unable or unwilling to provide accurate observations of demented patients' behavior, and thus are incapable of assisting with this important research. This study examined a process by which nurse aides were enlisted to identify and rate agitation in late stage dementia patients.
\end{abstract}

Ann L. Whall, PhD, RN, FAAN, School of Nursing, The University of Michigan, Ann Arbor, Michigan.

Margaret E.A. Black, PhD, RN, School of Nursing, McMaster University, Hamilton, Ontario, Canada.

Dawn J. Yankou, PhD, RN, Faculty of Health Sciences, University of Western Ontario, London, Ontario, Canada.

Carla J. Groh, PhD, RN, College of Health Professions, University of Detroit Mercy, Detroit, Michigan.

Barbara J. Kupferschmid, MSN, RN, School of Nursing, The University of Michigan, Ann Arbor, Michigan.

Norman L. Foster, MD, Department of Neurology, School of Medicine, The University of Michigan, Ann Arbor, Michigan

Roderick Little, PhD, Department of Biostatistics, School of Public Health, The University of Michigan, Ann Arbor, Michigan.
Results indicate that nurse aides accurately identified agitation at a high level of agreement $(r=>.90)$, on three occasions, with nurse experts. This high level of agreement was achieved along with a high level of nurse aide participation (75 percent), and with relatively little training time, i.e., approximately one hour per nurse aide. There were no significant differences in the demographic characteristics of aides participating versus those declining participation; likewise, participation rates in unionized versus non-unionized homes were not significantly different. The characteristics of the training program are described and the opinions of both nurse aides and administrators discussed as to why this program was successful.

\section{Introduction}

Although nurse aides give the majority of care in nursing homes, there is little research on the enlistment of nurse aides in the accurate identification of onset and level of agitation in nursing home residents. ${ }^{1-3}$ Agitation increases in frequency as dementia progresses and unrecognized and untreated agitation may develop into a state of acute aggression (termed catastrophic reaction), in which the demented person becomes severely disturbed and may injure themselves and others. ${ }^{3}$ As persons in late stage dementia are often unable to speak and describe their needs, accurate and ongoing identification of agitation by nurse aides is particularly important. ${ }^{4}$

The ability of nurse aides' to validly and reliably identify and quantify the onset and level of agitated behavior 
in dementia patients was the focus of this study. Adult learning principles were used to design a short term training program for the accurate identification and quantification of agitation. The research questions addressed whether acceptable validity and reliability could be achieved with relatively brief training of nurse aides, and if nurse aides were interested in participating in such training. A nursing care routine closely associated with agitation (the shower bath) was chosen as the event in which nurse aides' rating of agitation could be compared to that of expert nurses.

\section{Methods}

\section{Sample}

Three categories form the sample - participating nursing homes and nurse aides, and the patients being rated. Five nursing homes were selected from 17 homes within one nursing home corporation. The five homes were selected based upon homogeneity of location and patients, and of nurse aides across homes. In addition, the selected homes had a relatively large staff of nurse aides employed for more than one year. Agency administrators had indicated that nurse aides employed longer than one year usually exhibited sustained commitment to the nurse aide role; thus, these aides were approached for participation in the study. The homes were located in working class, suburban communities of a large Midwestern city; Medicaid was the most common source of funding for residents. Two of the nursing homes were unionized and three were not. All homes were certified as Medicaid and Medicare eligible and all were approved and licensed by the State Department of Public Health. Nurse aides in these homes received the same basic nurse aide training which met all state and federal guidelines.

Nurse aides who worked on the day shift, and had been employed for over one year, were identified by the administration - the study was explained to these aides and human subject safeguards carried out. The nurse aides were not promised any financial remuneration or special work assignments, but were promised a letter explaining their participation in the study. It was suggested that they use this letter both for their current personnel file and as an addition to their resume materials. In group discussions, most aides who agreed to participate, stated that they saw the study as an opportunity to enhance their training history, as well as an opportunity to increase the variety in their work.

Out of the 20 nurse aides approached, 75 percent of the total (15) agreed to participate after individual and group explanations were made; (two later dropped out due to scheduling problems in the nursing home). Of the remaining 13 aides, nine were from unionized homes and four were from non-unionized homes. There was no significant difference (Chi-square) in the refusal rate by nurse aides employed in unionized versus non-unionized homes or on other demographic characteristics of nurse aides such as race. Most aides were female, had been employed at the home an average of four years, and were almost equally divided between African-American and Caucasian (see Table 1).

The dementia patients $(\mathrm{N}=31)$ had a verified diagnosis of either Alzheimer's disease (AD) or multi-infarct dementia or a mixture of the two conditions. Patient criteria were:

- Demonstrated agitation at least half the time during personal care;

- A score of five or less on the Mini-MentalStatus Exam;

- No current acute health condition such as pneumonia; and

- English as their primary language.

Relatives of eligible patients were sent letters describing the study and asking permission for their family member to be approached to participate. When relatives gave permission, the study was explained in simple terms to the demented patients and their verbal assent sought. Refusal by relatives (18 percent) was usually related to relatives' wishing to spare their family member, e.g., "they have been through enough."

\section{Procedure}

Through agreement with the nursing home, the nurse aide training for the study was used to satisfy a small portion of their required in-service education. The training program for the nurse aides was developed using a synthesis of adult learning theories. Adult learning principles derived from these theories emphasize active participation of the learner, the use of learner input, immediate feedback to the learner, and demonstration of procedures followed by return demonstration by the learner. These theories suggest that when adults are active participants in planning and modifying aspects of their learning, interest in and cooperation by the participants is enhanced.

Audiovisual tapes (which are commercially available) were used to demonstrate the onset and levels of various types of agitation in dementia patients. In this descriptive exercise, nurse aides were asked to identify the onset 
Table 1. Nurse aide characteristics by nursing home

\begin{tabular}{|c|c|c|c|c|c|}
\hline \multicolumn{6}{|c|}{ Nursing homes } \\
\hline Characteristics & One & Two & Three & Four & Five \\
\hline \multicolumn{6}{|l|}{ Gender } \\
\hline Male & 1 & 0 & 1 & 0 & 0 \\
\hline Female & 5 & 2 & 1 & 2 & $2^{*}$ \\
\hline \multicolumn{6}{|l|}{ Race } \\
\hline African American & 3 & 0 & 2 & 2 & 0 \\
\hline Caucasian & 3 & 1 & 0 & 0 & 2 \\
\hline Hispanic & 0 & 1 & 0 & 0 & 0 \\
\hline \multicolumn{6}{|l|}{ Length of employment } \\
\hline Range & $1-4.5$ & $4-17$ & $2-3$ & $2.5-4$ & 2 \\
\hline Mean & 3.3 & 10.5 & 2.5 & 3.3 & 2.0 \\
\hline
\end{tabular}

and the level of agitation displayed on the videotapes; the nurse experts used explanations and corrected nurse aides' ratings as needed; when there was a rating conflict, the tape was re-rated until agreement between aide and expert was established. The nurse aides were able to stop the tapes and ask questions as needed. Their suggestions were solicited when it was appropriate to do so throughout the rating of the tapes and throughout the training procedure.

In the first 30-minute training session, the nurse aides were introduced to identification of agitation on the tapes, using the Cohen-Mansfield Agitation Inventory (CMAI). ${ }^{5}$ In the second 30 -minute training session, the nurse experts and nurse aides independently rated the tapes, and the tape ratings were compared and discussed until 80 percent agreement between the aides and nurse experts was achieved. The nurse aides' understanding and use of the CMAI to rate agitation was evaluated at several points throughout the session, with an emphasis on understanding why any difference occurred in ratings of the aides versus the experts. Non-participant Observation Principles (NPO) were also discussed for use during the shower bath observations. In the NPO technique the observer actively holds to a minimum all interaction with anyone or anything other than the direct observation of the subject.

The nurse aides were paired with nurse experts to jointly observe on three occasions a shower bath, (approximately 10 minutes), for each of the study patients. Both the nurse aide and the nurse expert made brief notes during observations of the shower bath and immediately completed and totaled the CMAI upon bath completion. The first of the three observation/rating sessions was designed to be interactive in nature, i.e., the nurse expert/nurse aide pair discussed questions concerning their observations and CMAI ratings during this shower bath; these discussions were designed to correct any problems in the actual bath situation. The second and third observations were not interactive and each nurse expert/nurse aide pair completed the CMAI immediately 
Table 2. Measures of agreement between ratings of nurse aides and nurse experts

\begin{tabular}{|c|c|c|c|}
\hline Time period & $\begin{array}{c}\text { Pearson correlation of aide } \\
\text { and nurse expert ratings }\end{array}$ & $\begin{array}{c}\text { T-test for mean difference } \\
\text { in aide and nurse } \\
\text { expert ratings }\end{array}$ & $\begin{array}{c}\text { Prediction interval for } \\
\text { difference between aide } \\
\text { and nurse expert ratings }\end{array}$ \\
\hline One & 0.97 & $-2.19(\mathrm{p}=0.04)$ & $(-3.96,5.90)$ \\
\hline Two & 0.93 & $-0.41(\mathrm{p}=0.69)$ & $(-4.29,4.62)$ \\
\hline Three & 0.95 & $-0.72(\mathrm{p}=0.48)$ & $(4.08,4.67)$ \\
\hline
\end{tabular}

after the bath without consultation. All CMAI forms were removed from the nursing home, totaled and retotaled prior to data analyses.

\section{Measurement}

The Cohen-Mansfield Agitation Inventory was used to capture the onset and level of agitation during the shower baths. ${ }^{5,6}$ The CMAI was originally designed to identify agitation via retrospective recall of nurses and caregivers. The CMAI using this approach had an inter-rater reliability of .88 and in a later study with 408 nursing home residents, inter-rater reliabilities were $.92, .92, .88 .{ }^{6}$

In 1991 the CMAI was modified by Whall, et al, for use as a direct observational tool, that is, for use during a distinct nursing care procedure; this modification eliminated the retrospective recall format. ${ }^{7}$ The items on the CMAI, however, remained the same, only the format was changed. The interrater reliability in the initial trial of this new format, ranged from .72 to .81 in the three observations. ${ }^{7}$ The agreement of this modified CMAI was .80 with the Ward Behavior Inventory, indicating concurrent validity with an older instrument. The modified or direct observational format of the CMAI was used in this study.

\section{Findings}

Individual CMAI Scores averaged over the two raters ranged from 0 to 31 at time one (mean $=11.12), 0$ to 21 at time two $($ mean $=8.23)$ and 1 to 24 at time three $($ mean $=$ 7.38). The Pearson correlation coefficient $(p \leq .01)$ of the aide and nurse experts' ratings varied from 0.97 at time one, to 0.93 at time two, and 0.95 at time three. The correlation coefficient was higher at time one when some discussion between the nurse aide and nurse expert was permitted, and decreased somewhat when ratings were independent at times two and three.

Altman criticized the use of the Pearson correlation for measuring inter-rater agreement, and accordingly, Tab1e 2 describes two other aspects of rater agreement. ${ }^{8}$ The second column identifies whether the mean differences between raters are significantly different from zero for each time period. A significant difference is evidence that the raters differed in their average rating scores. No statistically significant differences were found at time two $(\mathrm{t}=-0.41, \mathrm{p}=0.69)$ and time three $(\mathrm{t}=-0.72, \mathrm{p}=$ 0.48 ), but there was a significant difference between the raters at time one $(t=-2.19, p=0.04)$, probably reflecting the novice status of the nurse aides at that time. By time two and three, however, the aides had most of their questions answered and their ratings were better calibrated with those of the nurse experts. The prediction intervals in Table 2 provide the range of values within which the differences in the ratings of the aide and expert are expected to lie within 95 percent confidence level. These limits narrow somewhat between the first time point and last two time points but are still quite substantial, suggesting that the CMAI scores of individual raters can differ by as much as four points.

\section{Discussion}

Conventional wisdom suggests that nursing home research is hindered because relatively untrained nurse aides are unable and/or unwilling to participate meaningfully in research activities; this study does not support this position. Rather, we found that nurse aides who worked for a year or more in nursing homes, were willing participants in research and made accurate observations when they understood the usefulness of a training program to them personally, and when adult learning principles were 
used in their training. Moreover, these results were achieved with relatively little nurse aide training (approximately one hour of total training per nurse aide).

After completion of the study, discussions were held with both the nurse aides and the nursing home administrators. At the outset of the project, the assistance of the vice president of the corporation was sought and had been given. The administrators identified that when questions arose as to the need to change nurse aide schedules so that the aides might remain on the project, this support facilitated their follow through. The administrators also believed that the success in recruiting and retaining of nurse aides to the study was based upon several features, one of which was trust. The nurse aides came to believe they could trust the researchers when (as promised) a nurse aides' refusal to participate in the study was not revealed to administration.

The nurse aides reported that the letters of recognition were tangible evidence of their effort and also reported they found the study helpful in their care of persons with dementia. In addition, the nurse aides reported that personal recognition was implied by their being accepted to participate in a "university" project. Since the training of nurse aides was pre-arranged with administration to count as a portion of the aide's required in-service education, the nurse aides also believed that this endorsement by the nursing home administration enhanced the status of the study. Finally, the nurse aides reported that the study was an interesting change of pace for them and an opportunity for variety in routine; aides reported that such opportunity to introduce variety into the routines, was rare in nursing homes.

\section{Conclusion}

In summary, key issues in the successful recruitment and training of nurse aides to identify and rate agitation in demented patients were:

- The recruitment of nurse aides with over one year of experience in nursing homes;

- The use of adult learning principles in the training of nurse aides which stressed their input into training;
- Recognition of the nurse aides' participation through letters and certificates; and

- The early enlistment of a nursing home officer to support the project.

The limitations of this study include the fact that the nursing homes and nurse aides were not randomly selected and were not entirely blind to the study purposes.

The results of this study are supportive of nurse aides carrying-out accurate observations of agitation in persons with dementia; such findings enhance the feasibility of research into interventions for early prevention and treatment of catastrophic reactions. The assistance of nurse aides is important, for research into this troubling behavior to some extent depends upon the ability and willingness of nurse aides to assist with rating intervention outcomes.

\section{Acknowledgment}

Funding for this project was provided by the National Institute of Nursing Research, F33 NR 06911-01; The University of Michigan Office of Vice President for Research; and the National Institutes of Health, P50-AG0871 (MADRC).

\section{References}

1. Hartig M, Engle V, Graney M: Accuracy of nurse aides' functional assessments of nursing home residents. Journal of Gerontology. 1997; 52A(3): M142-M148.

2. Spore DL, Smyer MA, Cohn MD: Assessing nursing assistants' knowledge of behavioral approaches to mental health problems. The Gerontologist. 1991; 31(3): 309-317.

3. Whall A, Gillis G, Yankou D, et al: Disruptive Behavior in elderly nursing home residents, Journal of Gerontological Nursing. 1992; 18(10): 13-19.

4. Simmons S, Schnelle J, Uman G, et al: Selecting nursing home residents for satisfaction surveys. The Gerontologist. 1997; 37(4): 543550 .

5. Cohen-Mansfield J, Billig N: Agitated behaviors in the elderly. Journal of the American Geriatrics Society. 1986; 34: 711-721.

6. Cohen-Mansfield J, Marx MS, Rosenthal AS: A description of agitation in a nursing home. Journal of Gerontology. 1989; 44(3): 77-84.

7. Chrisman M, Tabar D, Whall A, Booth D: Agitated behavior in cognitively impaired elderly. Journal of Gerontological Nursing. 1991; 17(12), 9-13.

8. Altman DG: Practical statistics for medical research. London: Chapman and Hall. 1991. 